\title{
GESTACIÓN CUÁDRUPLE ESPONTÁNEA. UN DESAFÍO PARA EL EQUIPO MULTIDISCIPLINARIO EN UN SERVICIO DE SALUD
}

\author{
Eduardo Salgado M. ${ }^{1}$, José Lattus O. ${ }^{1}$, Verónica Barrera C. ${ }^{1}$, Hugo Salvo M. ${ }^{2}$, Margarita \\ Mena G. ${ }^{3}$, Carolina Farías P. ${ }^{a}$, Daniel Aravena A. ${ }^{a}$, Ana Fritis L. ${ }^{a}$, Fancy Gaete V. \\ ${ }^{1}$ Servicio de Obstetricia y Ginecología, ${ }^{2}$ Servicio de Neonatología, ${ }^{3}$ Servicio de Anestesiología, ${ }^{4}$ Servicio de Anatomía \\ Patológica, Hospital Dr. Luis Tisné Brousse, Universidad de Chile, Campus Oriente.
}

a Internos de Medicina, Universidad de Chile.

\section{RESUMEN}

Damos a conocer un caso de gestación cuádruple espontánea, el manejo y su resolución.

\section{PALABRAS CLAVES: Gestación cuádruple, manejo y resolución}

\section{SUMMARY}

A clinical report of spontaneous quadruplet pregnancy is presented, and the management and outcome.

\section{KEY WORDS: Quadruplet pregnancy, management and outcome}

\section{INTRODUCCIÓN}

La frecuencia de embarazos multifetales (EM) ha aumentado considerablemente durante las últimas dos décadas y presentan un alto riesgo materno-perinatal el que es proporcional al número de fetos en gestación (1-6). El EM constituye alrededor del $3 \%$ del total de embarazos, siendo la frecuencia de gestación doble de $1 / 90$, el triple de $1 / 9.000$ y cuádruples (EMC) de 1/500.000 recién nacidos. Es así como en Latinoamérica (LA) la tasa de EM es alrededor de 28 a $29 \%$ como consecuencia de la reproducción asistida, siendo en el Reino Unido (RU) de $24 \%$. Respecto de la tasa de EM extremo (tres o más fetos), en LA es de $10,4 \%$, y en el RU inferior al $1 \%$, lo que se explica por el destino de los embriones pre implantacionales que quedan supeditados al ejercicio de la autonomía de los progenitores (7). Las técnicas de reproducción asistida elevan la inci- dencia, y en consecuencia la morbimortalidad materna y perinatal (1). La incidencia de EM asociada a inducción de la ovulación con clomifeno es aproximadamente de $7-10 \%$ para embarazos dobles, $1-5 \%$ para triples y $3 / 1000$ para cuádruples. Si se utiliza gonadotropinas la incidencia puede alcanzar el 30\% (8-12). El alto riesgo perinatal de la gestación múltiple se resume en la Tabla I (1).

El objetivo de esta comunicación es dar a conocer el manejo materno y perinatal de un caso clínico de embarazo cuádruple espontáneo, resuelto en la maternidad del Hospital Santiago Oriente.

\section{CASO CLÍNICO}

Paciente de 39 años, proveniente de la ciudad de Los Andes. Sin antecedentes mórbidos de importancia. Multípara de 3 , último parto en febrero de 2001. Usuaria de dispositivo intrauterino (DIU) 
Tabla I

MORBIMORTALIDAD PERINATAL EN GESTACIÓN MÚLTIPLE

\begin{tabular}{lccc}
\hline Variable & Dobles & Triples & Cuadrúples \\
\hline Peso promedio (g) & 2.347 & 1.687 & 1.309 \\
Edad gestacional promedio al parto (s) & 35,3 & 32,2 & 29,9 \\
RCIU (\%) & $14-25$ & $50-60$ & $50-60$ \\
Ingreso a UTI (\%) & 25 & 75 & 100 \\
Promedio de estadía en UTI (días) & 18 & 30 & 50 \\
Handicap mayor (\%) & - & 20 & 50 \\
Riesgo de parálisis cerebral comparados con únicos & 4 veces & 17 veces & - \\
Riesgo de muerte infantil & 7 veces & 20 veces & - \\
\hline
\end{tabular}

Fuente: American College of Obstetrician and Gynecologists Committee on Practice Bulletins-Obstetrics. Multiple gestation: complicated twin, triplet, and high-order multifetal pregnancy. Obstet Gynecol. 2004 Oct; 104 (4): 869-83.

tipo Lippes desde 2001. Consulta en su ciudad por amenorrea de tres meses (FUR: 15/10/2003), el examen sugiere gestación en evolución y ausencia de señuelos del DIU. La ecografía demuestra gestación cuádruple de 12 semanas y ausencia de DIU y es derivada a la unidad de alto riesgo obstétrico local (ARO). El ultrasonido efectuado a las 25 semanas revela gestación cuádruple, fetos I y II femeninos, monocorial-monoamnióticos; fetos III y IV masculinos, monocorial, biamniótico. Fetos I, II y III creciendo en percentil 25, feto IV en percentil 50. Líquido amniótico en volumen normal. Anatomía de los fetos sin alteraciones evidenciables, doppler materno-fetal normal.

A las 28 semanas, es referida a Santiago para la resolución del embarazo en un servicio especializado de neonatología. El 11 de mayo de 2004 ingresa a la Unidad de ARO del Hospital Santiago Oriente, cursando una gestación cuádruple de

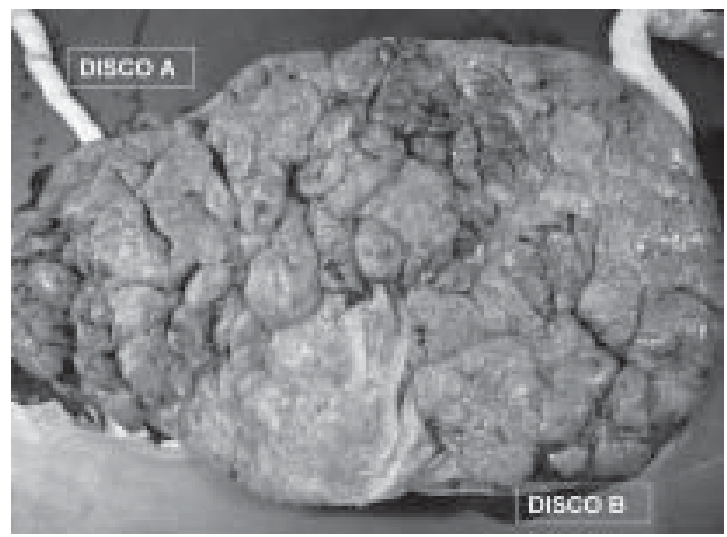

Figura 1. Discos placentarios de placenta de embarazo cuádruple, inserción materna.
29+6 semanas. Al ingreso se constata síndrome emético con deshidratación moderada de 4 días de evolución y síndrome hipertensivo del embarazo de inicio reciente. Se realiza video endoscopia digestiva alta el 12/05/2004, que informa erosiones del tercio distal del esófago, cubiertas por fibrina, estómago con mucosa levemente eritematosa, píloro ampliamente permeable, duodeno normal hasta la segunda porción, no se realizó biopsia; conclusión: erosiones ulceradas de esófago distal. Se inicia tratamiento con ranitidina $150 \mathrm{mg}$ cada 12 horas, por vía oral. El fondo de ojo revela papilas de bordes netos, vasos normales, sin hemorragias ni exudados. LDH $522 \mathrm{U} / \mathrm{l}$, fosfatasas alcalinas $412 \mathrm{U} / \mathrm{l}$, hematocrito $42,6 \%$, hemoglobina $13,4 \mathrm{~g} / \mathrm{dl}$, plaquetas $418 \mathrm{~K} / \mathrm{ul}$, leucocitos $13,2 \mathrm{~K} / \mathrm{ul}$, resto de los exámenes en rangos de normalidad. Evoluciona con presión arterial diaria entre 149/91 y $111 / 51$, asintomática.

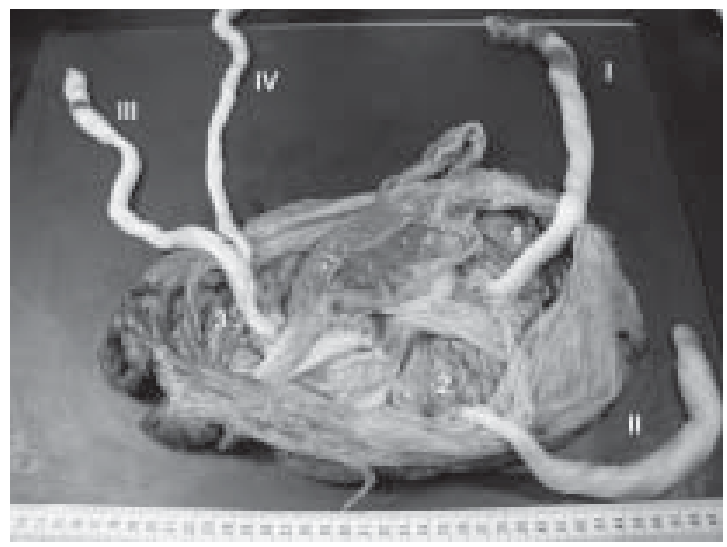

Figura 2. Placenta de embarazo cuádruple, 3 sacos $(1,2,3)$, uno monoamniótico, y cuatro cordones (I-II-IIIIV). 


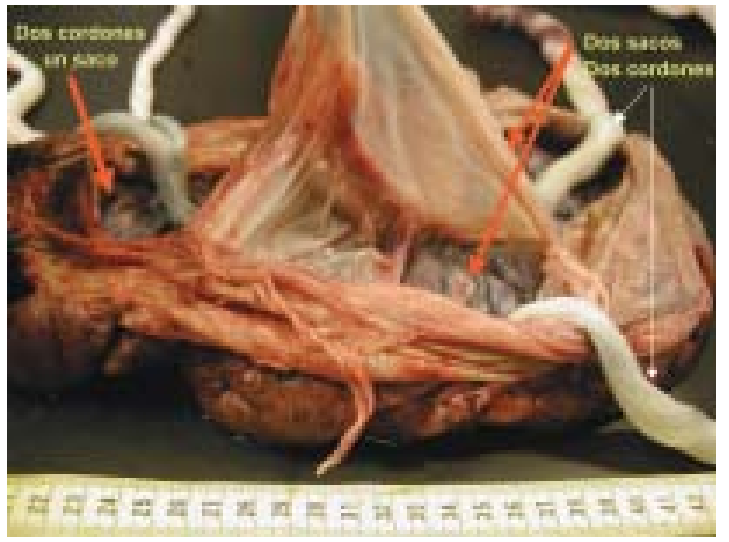

Figura 3. Placenta de embarazo cuádruple, 3 sacos, cuatro cordones.

La ecografía efectuada a las $28+6$ semanas muestra feto I de $1.158 \mathrm{~g}$, feto II de $1.229 \mathrm{~g}$, feto III de $1.233 \mathrm{~g}$ y feto IV de $928 \mathrm{~g}$ en percentil 5-10 con doppler de arteria umbilical normal y líquido amniótico normal; cuello uterino de $28 \mathrm{~mm}$ de longitud. La ecografía efectuada a las $29+6$ semanas muestra feto I de $1.373 \mathrm{~g}$ en percentil $10-25$, feto II de $1.182 \mathrm{~g}$ en $10-25$, feto III de $1.330 \mathrm{~g}$ en percentil $10-25$ y feto IV de $980 \mathrm{~g}$ bajo el percentil 5 , líquido amniótico normal y doppler materno fetal normal. La ecografía efectuada a las 32 semanas muestra feto I de $1.662 \mathrm{~g}$ en percentil 10, feto II de $1.779 \mathrm{~g}$ en percentil $10-25$, feto III de $1.703 \mathrm{~g}$ en percentil $10-25$ y feto IV de $1.273 \mathrm{~g}$ bajo el percentil 5, líquido amniótico normal, doppler revela resistencia arteria umbilical elevada en feto III. Doppler fetal normal en los 4 fetos a las 33 semanas y $33+4$ semanas.

Por el compromiso materno, dado el gran volumen abdominal y síndrome hipertensivo, se decide interrumpir el embarazo mediante operación cesárea electiva la que se efectúa el 4 de junio de 2004. Se extrae feto I en podálica, femenino, $1.675 \mathrm{~g}$, Apgar 9-9; feto II en podálica, femenino, $1.810 \mathrm{~g}$, Apgar 8-9; monocoriales, monoamnióticos. Feto III en podálica, masculino, $1.785 \mathrm{~g}$, Apgar 8-8; feto IV en podálica, masculino, $1.310 \mathrm{~g}$, Apgar 8-9, pequeño para la edad gestacional; bicoriales, biamnióticos. Esterilización tubaria según técnica de Pomeroy clásica. Evolución materna con puerperio fisiológico, normo tensa, es dada de alta al $4^{\circ}$ día.

Informe anátomo-patológico de las placentas (№ 403106): Placentas de embarazo cuádruple. Se identifican dos discos placentarios fusionados en forma laxa. Uno de los discos, A es monocorial,

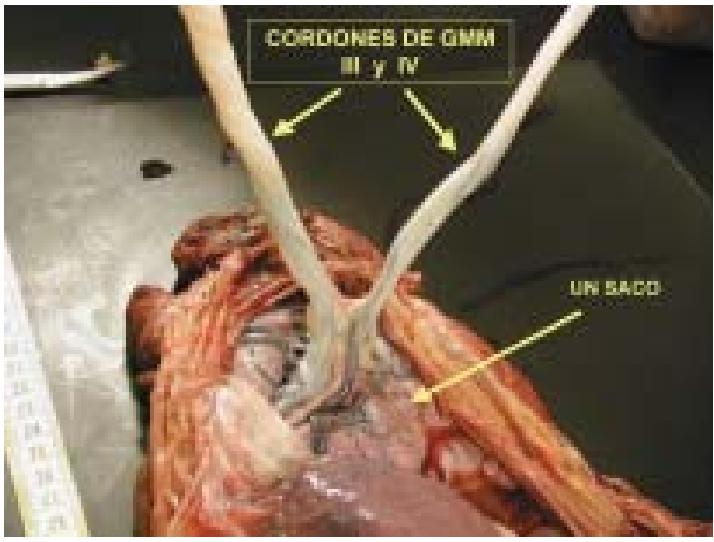

Figura 4. Embarazo cuádruple, placenta y cordones de monocorial-monoamniótico con base común de inserción.

monoamniótico, con la inserción de los dos cordones en forma central, separados por $0,2 \mathrm{~cm}$, de 23 y $25 \mathrm{~cm}$ de largo, ambos con tres vasos. Membranas opacas. Peso 423g (Figuras 1, 2, 3). El segundo disco $\mathrm{B}$ con dos cavidades amnióticas, con membranas que separan la placenta en dos áreas desiguales, 60 y $40 \%$ respectivamente. Cordones ligeramente excéntricos, de 30 y $24 \mathrm{~cm}$, respectivamente, ambos con tres vasos. Peso $525 \mathrm{~g}$. Disco A, placenta gemelar monocorial, monoamniótica, en percentil 10 para 33 semanas, inmadura, signos sugerentes de hipertensión arterial, cordones centrales con tres vasos (Figura 4). Disco B, placenta gemelar bicorial, biamniótica, fusionadas, en percentil 10 para 33 semanas, con proporción de 60 y $40 \%$ respectivamente, inmaduras, signos sugerentes de hipertensión arterial.

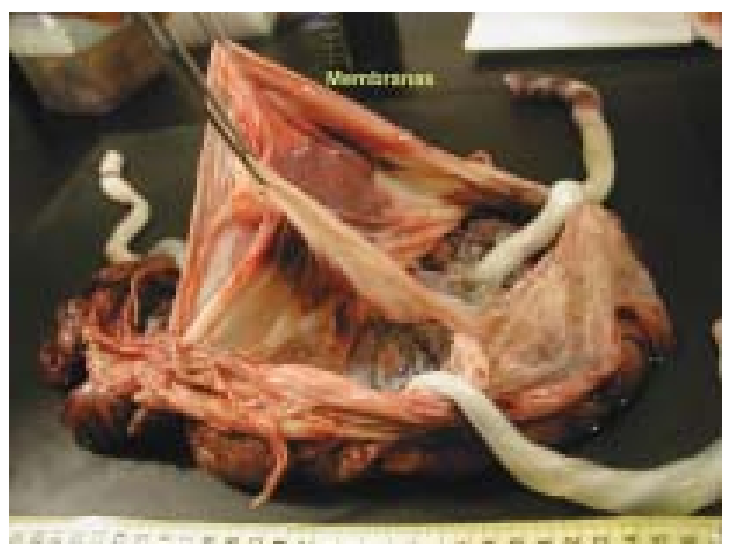

Figura 5. Placenta de embarazo cuádruple, tres sacos, membranas amnióticas y cordones. 


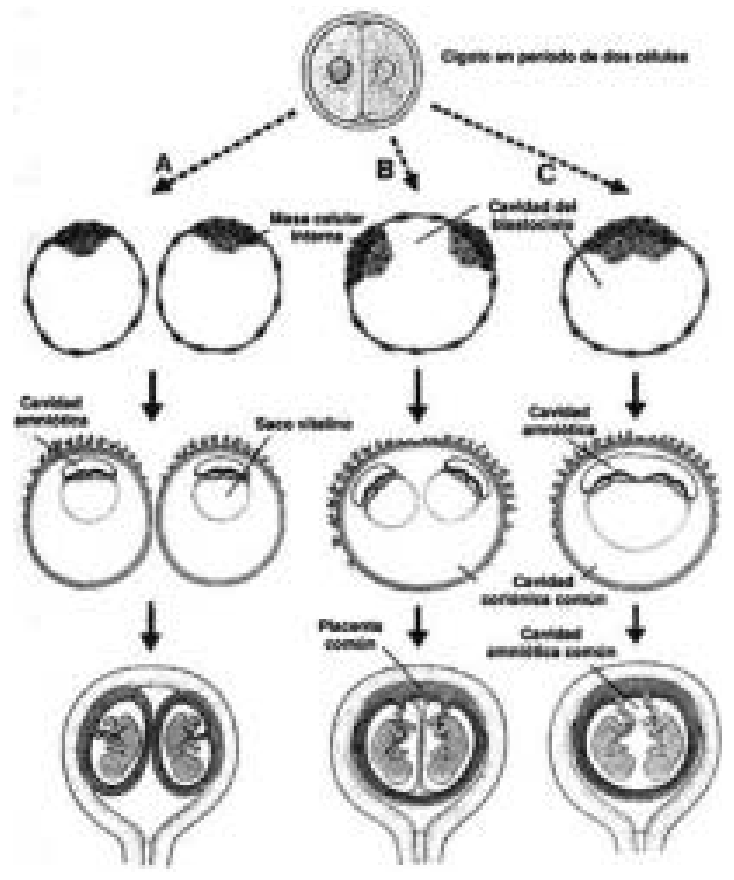

Figura 6. Formación de gemelos univitelinos: A) biocoriales-biamnióticos. B) monocoriales-biamnióticos. C) monocorial-monoamniótico.

Cordones de inserción excéntrica con tres vasos (Figura 5). No se observa signos de corioamnionitis.

\section{Evolución de los recién nacidos}

Gemelo I: femenino, $1.675 \mathrm{~g}$ buena evolución, 8 días de UTI y 10 de intermedio, total 21 días, membrana hialina leve, con requerimiento de oxígeno, requirió 1 dosis de surfactante, CPAP 24 horas, alimentándose VO, peso al alta $2.065 \mathrm{~g}$. Ecografía encefálica normal. Diagnóstico: Gemela I de IV, RN pretérmino 33 PEG, ictericia del prematuro, al alta 36 semanas EG corregida.

Gemelo II: femenino, $1.810 \mathrm{~g}$, síndrome de distrés adaptativo (SDA) y pequeño neumomediastino. Requirió oxígeno fi $\mathrm{O}_{2}$ 0,27- 0,23 por 24 h, 8 días de UTI y 3 de intermedio, total 19 días. Actualmente alimentándose $\mathrm{VO}$, peso al alta $2.055 \mathrm{~g}$. Ecografía encefálica normal. Diagnóstico: Gemela II de IV, RN pretérmino 33 semanas, SDA, ictericia del prematuro.

Gemelo III: masculino, $1.785 \mathrm{~g}$ buena evolución sin requerimiento de oxígeno, alimentándose VO, 9 días en UTI, 4 en intermedio, total 19 días. Peso al alta $2.150 \mathrm{~g}$. Ecografía encefálica normal.
Diagnóstico: Gemelo III de IV, RN pretérmino, 33 semanas, SDA, ictericia del prematuro.

Gemelo IV: masculino, $1.310 \mathrm{~g}$, buena evolución sin requerimiento de oxígeno, alimentándose por sonda, 11 días en UTI, 22 en intermedio, total 33 días. Peso de alta $2.050 \mathrm{~g}$. Hemangioma cavernoso parietal derecho. Ecografía encefálica normal. Diagnóstico: Gemelo IV de IV, RN de pretérmino pequeño para la edad gestacional, 33 semanas, ictericia del prematuro, hemangioma cavernoso parietal derecho.

\section{DISCUSIÓN}

La frase "gestaciones múltiples - problemas múltiples" $(1,7)$, toma su real dimensión cuando el equipo multidisciplinario se enfrenta a EM extremo con tres o más fetos (8-17), que actualmente ha aumentado extraordinariamente con el avance de la ciencia y la tecnología en el tratamiento de la infertilidad (1-6, 8-12). No se considera raro entonces hoy día los EM, y que sólo en EEUU haya más de 130.000 al año, por lo que se han presentado estrategias de manejo, basadas en evidencias, que ayudan al clínico a resolver estos complicados casos clínicos (1, 13-25). Desde 1980 la frecuencia de embarazo gemelar se ha incrementado en $65 \%$, y los triples y EMC o más, en $500 \%$ (1), así como también han aumentado los riesgos maternos y perinatales asociados a embarazos multifetales.

El caso clínico que damos a conocer queda fuera de la problemática que se menciona en la bibliografía mundial, ya que es una gestación cuádruple sin que mediara tratamiento con gonadotropinas o programa de fecundación in vitro, y con resultado de gemelos monozigóticos, unas bicoriales-biamnióticos y los otros monocorialesmonoamnióticos.

Hacemos notar que los gemelos monocorialesmonoamnióticos fueron diagnosticados en la ecografía de las 25 semanas en Los Andes, lo que revela la excelencia de los médicos regionales. Este tipo de GMM de etiología desconocida (26), monozigóticos, y como consecuencia de la bipartición del huevo durante o después de la diferenciación de la placa embrionaria, diferenciación inicial del blastómero, después de la formación del amnios y antes de la implantación del huevo en el útero, entre el día 7 y 13 , constituye el 1 a $2 \%$ de los embarazos gemelares (Figura 6). Se informa de una alta morbilidad y mortalidad después de las 32 semanas, cercana al 50 a $62 \%$, por las complicaciones asociadas como transfusión inter- 
gemelar, malformaciones congénitas, restricción del crecimiento intrauterino, 40 a $70 \%$ de patología funicular como nudos, enroscamientos o enredos de los cordones umbilicales, y $15-20 \%$ de parto prematuro (27-36). El control de este tipo de gestación se debe realizar con monitorización electrónica seriada y ecografías sucesivas con estudio doppler de las arterias umbilicales. Por lo anterior un gran porcentaje se resuelve a las 32 semanas, para evitar el riesgo de muerte fetal intrauterina por enredos del cordón (26-37). La operación cesárea es la vía de resolución preferencial (1, 37), no obstante puede acontecer que se produzca un parto espontáneo (38).

En general según datos de la literatura la incidencia de gemelos monocigóticos es de $33 \%$ y dicigóticos de $66 \%$. La placentación en gemelos monozigóticos, monocorial-monoamniótica es de $1-5 \%$, monocorial-biamniótica $65 \%$ y bicorialbiamniótica de $30 \%$. El diagnóstico de corionicidad por ultrasonografía del primer trimestre tiene sensibilidad de $95 \%$; si se observan placentas claramente separadas, corresponde a gestación bicorial-biamniótica $(39,40)$. Entre la sexta y la décima semana de gestación el conteo del número de sacos corresponde al número de corion, sin embargo se puede subestimar en EM hasta en un $15 \%$ de los casos.

Dado que el amnios se diferencia después del corion, la bicorionicidad implica biamniocidad. El amnios se observa a partir de la 7 a 8 semanas. Hacia la décima semana las membranas amnióticas de un embarazo biamniótico se yuxtaponen, formando una membrana entre ellos. El signo de lambda podemos verlo en embarazos bicoriónicos con placentas fusionadas en que se visualiza una proyección triangular isoecoica con la placenta que se extiende desde la superficie placentaria hacia la membrana interfetal. La ausencia de este signo no excluye bicorionicidad ni implica monocorionicidad, la sensibilidad y especificidad es 94 y $88 \%$, respectivamente (1). La importancia de determinar amniocidad y corionicidad radica en la mortalidad fetal que varía según las variantes, a saber: bicorial-biamniótico un $5 \%$, monocorialbiamniótico un 10-20\%, monocorial-monoamniótica hasta un $50 \%$.

Haremos una breve mención de la reducción embrionaria, que en el país por su legislación respecto del aborto no está autorizada. Se realiza en gestaciones de alto orden (tres o más) para reducir el número de fetos (1 ó 2), disminuyendo de esta forma la morbimortalidad perinatal especialmente al prolongar la gestación $(1,41)$. Es útil en fetos con malformación grave para mejorar el pronóstico del gemelo normal. El procedimiento se realiza en el primer trimestre o inicio del segundo. La reducción embrionaria con coagulación con láser del sitio de inserción placentario del cordón tiene resultados controversiales y el riesgo materno y perinatal es importante.

Los EM dan cuenta de un 10 a $12 \%$ de las muertes perinatales, la mortalidad en gemelos es 4 veces mayor que la de los fetos únicos, y 6 veces en EM de 3 ó más fetos. La tasa de aborto al menos es el doble que en embarazo único (1). El óbito fetal, puede ocurrir en cualquiera de los fetos en un EM, con riesgo de coagulación intravascular diseminada, informada en el $25 \%$ de los casos, usualmente 3 a 5 semanas después (1, 20). Otra complicación es el síndrome del feto evanescente que corresponde a la desaparición de un gemelo en el primer trimestre (20\% EM), afortunadamente el otro gemelo no se afecta (20). En casos de óbito fetal el seguimiento con análisis semanal del sistema hemostático debe realizarse hasta la madurez pulmonar comprobada. En embarazos monocoriales cuando la muerte de un feto ocurre después de las 14 semanas la pérdida del tono vascular del feto muerto favorece la transfusión masiva de sangre desde el gemelo sobreviviente, dando como resultado isquemia aguda con muerte fetal o daño neurológico (encéfalomalacia multiquística) y necrosis cortical renal.

Respecto del síndrome de parto prematuro (PP), aproximadamente el $10 \%$ de los partos corresponde a gestaciones gemelares, en el grupo de EM es una complicación frecuente ocurriendo en $50 \%$ de los casos. En embarazo gemelar el riesgo es 5 veces más que en el de un embarazo único, para los EM triples el riesgo es 10 veces mayor (42).

En el caso clínico presentado destacamos el peso de los recién nacidos con un peso promedio de $1.700 \mathrm{~g}$, siendo habitual que los cuádruples no pesen en promedio más de $1.300 \mathrm{~g}$ (42), y la resolución a las 33 semanas +5 días, con un promedio de estadía de 20 días para los tres primeros y de 33 días para el de menor peso.

Entre las recomendaciones de la American College of Obstetrician and Gynecologists se menciona la cervicometría transvaginal (valores menor a $25 \mathrm{~mm}$ el riesgo es de $50 \%$ de PP entes de las 32 semanas) (1); la presencia de fibronectina fetal serviría para predecir PP, sin embargo, las evidencias no son suficientes como para incluirlo como examen de rutina (1). El uso de corticoides en EM tendría menor efecto en madu- 
ración pulmonar que en embarazo único, sin embargo, se recomienda su uso en dosis habituales (43). No se recomienda, la hospitalización, el reposo de rutina, el cerclaje profiláctico $(1,44)$ y la tocolisis profiláctica (1).

La rotura prematura de membranas (RPM), tiene una mayor frecuencia en el EM, el manejo es similar al de gestación única, según la normativa vigente (1).

Las malformaciones congénitas en EM presentan una incidencia de $5-10 \%$, y de estos un $50 \%$ de fetos gemelares muertos in utero (21). Las malformaciones son más frecuentes en embarazos monocoriales (50\% más que en bicoriales), generalmente son múltiples y letales. La frecuencia de malformaciones es el doble en embarazos gemelares y 4 veces más frecuentes en triples.

En la restricción del crecimiento intrauterino, el 12 a $14 \%$ son gemelares y más del $50 \%$ nacen pesando menos de $2.500 \mathrm{~g}(1,14,42)$.

En el síndrome de transfusión feto-fetal, el 15\% de los gemelos monocoriales presentan signos clínicos de transfusión intergemelar, con altas tasas de mortalidad fetal $(1,39)$. Pueden existir shunt arterio-venosos múltiples o únicos, donando un feto sangre al otro en forma permanente conduciendo a hidrops e hipervolemia del receptor $y$ anemia en el donante. El diagnóstico se sospecha por ser gestación monocorial, tamaño fetal dispar, polihidroamnios en el saco del feto mayor y oligoamnios en el pequeño. Si la prematurez extrema impide el parto se puede intentar la amnioreducción seriada o la anastomosis con ablación con láser por fetoscopia. El polihidroamnios se presenta en 5 a $8 \%$ de los EM, principalmente en monoamnióticos (34).

Una de las complicaciones maternas más frecuente es la preeclampsia, la que es 3 veces mayor en embarazos múltiples, es más precoz y grave; si es severa pueden acontecer complicaciones como síndrome HELLP u otros cuadros graves (1). Aproximadamente el $40 \%$ de gemelares y $60 \%$ de los trillizos se ven afectados por síndrome hipertensivo del embarazo; hay reportes que señalan que la reducción fetal puede disminuir en un $14 \%$ este riesgo $(1,45)$. La hemorragia obstétrica es significativamente de mayor frecuencia, habitualmente por inercia uterina debido a la sobre distensión uterina. Otras patologías como hígado graso agudo (46), tromboembolismo, coagulopatías, embolia pulmonar, prurigo urticarial de pápulas y placas del embarazo (47) y las estrías por el exceso de peso y la rápida distensión abdominal.

\section{CONCLUSIONES}

EI EM corresponde a una gestación de alto riesgo materno y perinatal, que exige un manejo multidisciplinario, con infraestructura de nivel terciario especialmente para la atención de los neonatos. Se aconseja hospitalización materna precoz, evaluación de la condición fetal seriada con doppler materno-fetal, seguimiento del crecimiento fetal ultrasonográfico, inducción de madurez pulmonar con corticoides según normativa e interrupción del embarazo desde las 32 semanas.

AGRADECIMIENTOS: a la Sra. Marjorie Berrios Segovia, por su aporte en la edición de texto e imágenes.

\section{BIBLIOGRAFÍA}

1. American College of Obstetrician and Gynecologists Committee on Practice Bulletins-Obstetrics; Society for Maternal-Fetal Medicine; ACOG Joint Editorial Committee. ACOG Practice Bulletin \#56: Multiple gestation: complicated twin, triplet, and high-order multifetal pregnancy. Obstet Gynecol 2004; 104(4): 869-83.

2. Gonen R, Herman E, Asztalos EV, et al. The outcome of triplet, quadruplet and quintuplet pregnancies managed in a perinatal unit: obstetric neonatal and follow up data. Am J Obstet Gynecol 1990; 162: 454-9.

3. Skrablin S, Kuvacic I, Pavicic D, Kalafatic D, Goluza T. Maternal neonatal outcome in quadruplet and quintuplet versus triplet gestations. Eur $\mathrm{J}$ Obstet Gynecol Reprod Biol 2000; 88: 147-52.

4. Malinowski W. Zaklad Perinatologii Institutu Ksztalcenia Medycznego, AS, Kielcach. Triplets, quadruplets and more. Ginekol Pol 2004; 75(11): 892-5.

5. Salihu HM, Aliyu MH, Kirby RS, Alexander GR. Effect of advanced maternal age on early mortality among quadruplets and quintuplets. Am J Obstet Gynecol 2004; 190(2): 383-8.

6. Arlettaz R, Paraskevopoulos E, Bucher HU. Triplets and quadruplets in Switzerland: comparison with singletons, and evolution over the last decade. $J$ Perinat Med 2003; 31(3): 242-50.

7. Massai R. Gestación múltiple. Medical Forum Internacional. Foro de Ginecología 2000; 3(1): 2-10.

8. Radivojevic K, Rosenkranz M, Deutinger J, Reinthaller A, Pollak A, Muller-Tyl E. Delivery of quadruplets after in vitro fertilization: perinatologic and ethical aspects. Wien Klin Wochenschr 1991; 103(23): 714-6.

9. Seoud MA. Toner JP, Kruithoff C, Muasher SJ. Outcome of twin, triplet and quadruplet in vitro fertilization pregnancies: the Norfolk experience. Fertil Steril 1992; 57: 825-34.

10. Seoud MA, Toner JP, Kruithoff C, Muasher SJ. 
Outcome of twin, triplet and quadruplet in vitro fertilization pregnancies: the Norfolk experience. Fertil Steril 1992; 57: 825-34.

11. Jonas HA, Lumley J. Triplets and quadruplets born in Victoria between 1982 and 1990. The impact of IVF and GIFT on rising birthrates. Med J Aust 1993; 17: 158(10): 659-63.

12. McGregor KK, Capone NC. Genetic and environmental interactions in determining the early lexicon: evidence from a set of tri-zigotic quadruplets. J Child Lang 2004; 31(2): 311-37.

13. Ron-El R, Caspi E, Schreyer P, et al. Triplet and quadruplet pregnancies and management. Obstet Gynecol 1981; 57: 458-63.

14. Skrablin S, Kuvacic I, Pavicic D, Kalafatic D, Goluza T. Maternal neonatal outcome in quadruplet and quintuplet versus triplet gestations. Eur $\mathrm{J}$ Obstet Gynecol Reprod Biol 2000; 88: 147-52.

15. Elliot JP, Radin TG. Quadruplet pregnancy: contemporary management and outcome. Obstet Gynecol 1992; 80: 421-4.

16. Tsenov D, Dacheva S. Choice of delivery method in multiple pregnancies: twins, triplets and quadruplets. Akush Ginekol (Sofiia) 2000; 39(3): 14-6.

17. Olatunbosum OA, Turnell RW, Sankaran K, Ninan A. Delayed interval delivery in quadruplets. Int Gynecol Obstet 1995; 50(3): 287-90.

18. Yamamori Y, Manou M, Sumiyoshi N, Kawachi S, Sawada K, Hasegawa K. Anesthetic management of caesarean section for the delivery of quadruplets. Masui 1991; 40(2): 301-5.

19. Brown DC, Massarelli E. Medical hypnosis and quadruplets: a case report. Am J Clin Hypn 2002; 45(1): 39-46.

20. Goldman GA, Dicker D, Feldberg D, Ashkenazi J, Yeshaya A, Goldman JA. The vanishing fetus. A report of 17 cases of triplets and quadruplets. J Perinat Med 1989; 17(2): 57-62.

21. Ryan RR, Wislocki GB. The birth of quadruplets, with an account of the placentas and fetal membranes. N Engl J Med 1954; 250(18): 755-8.

22. Mirsky AF, Bieliauskas LA, French LM, Van Kammen DP, Jonsson E, Sedvall G. A 39-year followup of the Genain quadruplets. Schizophr Bull 2000; 26(3): 699-708.

23. Auer C, Gromada KK. A case report of breastfeeding quadruplets: factors perceived as affecting breastfeeding. J Hum Lact 1998; 14(2): 135-41.

24. Mead LJ, Chuffo R, lawlor-Klean P, Meier PP. Breastfeeding success with preterm quadruplets. J Obstet Gynecol Neonatal Nurs 1992; 21(3): 221-7.

25. Rodis J, Vintzileos AM, Campbell WA, et al. Antenatal diagnosis and management of monoamniotic twins. Am J Obstet Gynecol 1987; 157: 1255-7.

26. Carr SR, Aronson MP, Coustan DR. Survival rates of monoamniotic twins do not decrease after 30 weeks gestation. Am J Obstet Gynecol 1990; 163: 719-22.

27. Dubeq F, Dufour Ph, Vinaitier D, et al. Monoamniotic twin pregnancies. Review of the literature, and a case report with vaginal delivery. Eur J Obstet Gynecol Reprod Biol 1996; 66: 183-6.

28. Rodis J, Mcliveen P, Egan J, et al. Monoamniotic twins: Improved perinatal survival with accurate prenatal diagnosis and antenatal fetal surveillance. Am J Obstet Gynecol 1997; 177(5): 1046-9.

29. Pijnenborg JM, Oei SG. The monoamniotic twin: a riskful event. Eur J Obstet Gynecol Reprod Biol 1999; 86(1): 51-3.

30. Overton T, Denbow M, Duncan K, Fisk N. First trimester cord entanglement in monoamniotic twins. Ultrasound Obstet Gynecol 1999; 13: 140-2.

31. Susuki S, Ishikawa G, Sawa R, et al. Umbilical venous pulsation indicating tight cord entanglement in monoamniotic twin pregnancy. J Ultrasound Med 1999; 18: 425-7.

32. Beasley E, Megerian G, Gerson A, Roberts NS. Monoamniotic twins: case series and proposal for antenatal management. Obstet Gynecol 1999; 93(1): 130-4.

33. Pedersen $\mathrm{MH}$, Larsen T. Three dimensional ultrasonography of monoamniotic twins. Uggeskr Laeger 2001; 163(5): 618-9.

34. Allen VM, Windrin R, Barrett J, Ohlsson A. Management of monoamniotic twin pregnancies: a case series of Toronto and Mount Sinai Hospital, Canada. BJOG 2001; 108(9): 931-6.

35. Suzuki S, Kaneko K, Shin S, Araki T. Incidence of intrauterine complications monoamniotic twin gestation. Arch Gynecol Obstet. Nippon Medical School Tokyo, Japan 2001; 265(2): 57-9.

36. Su LL. Monoamniotic twins: diagnosis and management. Acta Obstet Gynecol Scand 2002; 81(11): 9951000.

37. Runge HM, de Gregorio G, Hillemanns HG. Cesarean section in multiple pregnancies especially with triplets and quadruplets preparation and execution. Z Geburtshilfe Perinatol 1990; 194(5): 214-8.

38. Lattus J, Catalán A, Salvo H, Gaete F, Ibáñez CG, Rossel F. Gemelar monoamniótico. Una rareza como caso clínico en embarazos gemelares. Rev Chil Obstet Ginecol 2003; 68(6): 513-18.

39. Benirschke K, Kaufmann P: Pathology of the human placenta, 3rd Ed. New York: Springer-Verlag, 1995; 719-33.

40. Denbow M, Cox P, Taylor M, et al. Placental angioarchitecture in monochorionic twin pregnancies: Relationship to fetal growth, fetofetal transfusion syndrome, and pregnancy outcome. Am J Obstet Gynecol 2000; 182(2): 417-26.

41. Evans MI, Fletcher JC, Zador IE, et al. Selective first trimester termination in octuplet and quadruplet pregnancies: clinical and ethical issues. Obstet Gynaecol 1988; 71: 289-96.

42. Martin JA, Hamilton BE, Sutton PD, Ventura SJ, Menacker F, Munson ML. Births: final data for 2002. Natl Vital Stat Rep 2003; 52(10): 1-102.

43. Effect of corticosteroids for fetal maturation on 
perinatal outcomes. NIH Consens Statement 1994; 12: $1-24$

44. Flynn A, Scott F, Birrel W, Evans N. Delayed interval delivery in a quadruplet pregnancy: the use of transperineal ultrasound and cervical cerclage. Aust N Z J Obstet Gynaecol 1995; 35: 280-2.

45. Smith-Levitin M, Kowalik A, Birnholz J, Skupski DW, Hutson JM, Chevenak FA, et al. Selective reducción of multifetal pregnancies to twins improves outcome over nonreduced triplet's gestations. Am J Gynecol 1996; 175: 878-82.

46. Davidson KM, Simpson LL, Knox TA, D"Alton ME. Acute fatty liver of pregnancy in triplets gestation. Obstet Gynecol 1998; 91: 806-8.

47. Elling SV, Mckenna P, Powell FC. Pruritic urticarial papules and plaques of pregnancy in twin and triplet pregnancies. J Eur Acad Dermatol Venereol 2000; 14: $378-81$. 